\title{
ON SUBORDINATION RESULTS FOR CLASSES OF ANALYTIC FUNCTIONS WITH A CONVOLUTION STRUCTURE
}

\author{
J. K. PRAJAPAT and R. K. RAINA
}

\begin{abstract}
By adapting a familiar convolution structure of analytic functions, we define and investigate in this paper certain new classes of analytic functions. Among the various results studied (by using the methods of differential subordinations) are some of the useful properties and characteristics attributed to these function classes. Several consequences of the main results are considered and relevant connections with some known results are also pointed out.
\end{abstract}

\section{Introduction}

Let $\mathscr{A}_{p}$ denote the class of functions of the form

$$
f(z)=z^{p}+\sum_{k=p+1}^{\infty} a_{k} z^{k} \quad(p \in \mathbf{N}=\{1,2, \ldots\}),
$$

which are analytic and $p$-valent in the open unit disk $U=\{z ; z \in \mathrm{C}:|z|<1\}$.

For the functions $f$ and $g$ analytic in $\mathrm{U}$, we say that $f$ is subordinate to $g$ in $\mathrm{U}$, and write $f \prec g$, if there exists a function $w(z)$ analytic in $\mathrm{U}$ such that $|w(z)|<1, z \in \mathrm{U}$, and $w(0)=0$ with $f(z)=g(w(z))$ in $\mathrm{U}$. If $g$ is univalent in $\mathrm{U}$, then $f \prec g$ is equivalent to $f(0)=g(0)$ and $f(\mathrm{U}) \subset g(\mathrm{U})$.

If $f \in \mathscr{A}_{p}$ is given by (1.1) and $g \in \mathscr{A}_{p}$ is given by

$$
g(z)=z^{p}+\sum_{k=p+1}^{\infty} b_{k} z^{k},
$$

then the Hadamard product (or convolution) $f * g$ of $f$ and $g$ is defined (as usual) by

$$
(f * g)(z):=z^{p}+\sum_{k=p+1}^{\infty} a_{k} b_{k} z^{k} .
$$

Received 17 January 2008, in revised form 15 July 2009. 
Let $h$ be a convex function in $\mathrm{U}$ with $h(0)=1$ and $h(z)>0(z \in \mathrm{U})$. We denote by $\mathscr{H}_{p}^{m}(g ; \alpha, h)$ the class of functions $f(z)$ of the form (1.1) satisfying in terms of subordination the following condition for a given function $g(z) \in \mathscr{A}_{p}$ (defined by (1.2)):

(1.4) $(1-\alpha) \frac{r(p, m)(f * g)^{(m)}(z)}{z^{p-m}}+\alpha \frac{r(p, m+1)(f * g)^{(m+1)}(z)}{z^{p-m-1}} \prec h(z)$ $\left(\alpha>0, r(p, m)=\frac{(p-m) !}{p !}, p>m\left(m \in \mathrm{N}_{0}=\mathrm{N} \cup\{0\}\right) ; z \in \mathrm{U}\right)$.

In particular, if

$$
h(z)=\frac{1+A z}{1+B z} \quad(A \in \mathrm{C}, B \in[-1,0], A \neq B ; z \in \mathrm{U}),
$$

then for the purpose of the present investigation, the subclass $\mathscr{J}_{p}^{m}(g ; \alpha, A, B)$ $\left(\equiv \mathscr{H}_{p}^{m}\left(g ; \alpha, \frac{1+A z}{1+B z}\right)\right)$ of $\mathscr{A}_{p}$ of interest in this paper is obviously defined by

$$
\begin{aligned}
&(1-\alpha) \frac{r(p, m)(f * g)^{(m)}(z)}{z^{p-m}} \\
&+\alpha \frac{r(p, m+1)(f * g)^{(m+1)}(z)}{z^{p-m-1}} \prec \frac{1+A z}{1+B z}
\end{aligned}
$$

$\left(\alpha>0, r(p, m)=\frac{(p-m) !}{p !}, p>m\left(m \in \mathrm{N}_{0}=\mathrm{N} \cup\{0\}\right), A \in \mathrm{C}, B \in\right.$ $[-1,0], A \neq B ; z \in \mathrm{U})$, such that $\Re(A+B)>|A+B|, B \in(-1,0]$, or $-1 \leq \Re(A)<1, B=-1$; and if the parameters $A$ and $B$ in (1.5) satisfy the condition that $-1 \leq B<A \leq 1$, then we denote the subclass of $\mathscr{A}_{p}$ satisfying the inequality $(1.5)$ by $\mathrm{J}_{p}^{m}(g ; \alpha, A, B)$.

Also (for simplicity), we put

$$
\mathscr{J}_{p}^{m}\left(g ; \alpha, 1-\frac{2 \beta}{p-m},-1\right)=\mathscr{J}_{p}^{*}(g ; \alpha, \beta, m),
$$

where $\mathscr{J}_{p}^{*}(g ; \alpha, \beta, m)$ denotes the class of functions $f \in \mathscr{A}_{p}$ which satisfy the inequality

$$
\begin{aligned}
& \Re\left((1-\alpha) \frac{r(p, m)(f * g)^{(m)}(z)}{z^{p-m}}\right. \\
& \left.\quad+\alpha \frac{r(p, m+1)(f * g)^{(m+1)}(z)}{z^{p-m-1}}\right)>\frac{\beta}{p-m}
\end{aligned}
$$

$\left(\alpha>0,0 \leq \beta<p, r(p, m)=\frac{(p-m) !}{p !}, p>m\left(m \in \mathrm{N}_{0}\right) ; z \in \mathrm{U}\right)$.

The aforementioned function classes $\mathscr{J}_{p}^{m}(g ; \alpha, A, B)$ and $\mathscr{J}_{p}^{*}(g ; \alpha, \beta, m)$ contain several known classes of functions which have been studied in the 
recent past. For example, let the coefficients $b_{k}$ in (1.2) and the values of $m$ and $\alpha$ in (1.5) be, respectively, as follows:

$$
b_{k}=\frac{\left(\alpha_{1}\right)_{k-p} \ldots\left(\alpha_{q}\right)_{k-p}}{\left(\beta_{1}\right)_{k-p} \ldots\left(\beta_{s}\right)_{k-p}(k-p) !}, \quad m=0 \quad \text { and } \quad \alpha=\frac{p \lambda}{\alpha_{i}}
$$

$\left(\alpha_{j}>0(j=1, \ldots, q), \beta_{j}>0(j=1, \ldots, s), q \leq s+1 ; q, s \in \mathrm{N}_{0}=\right.$ $\mathrm{N} \cup\{0\})$, where the symbol $(\alpha)_{k}$ occurring in (1.8) is the familiar Pochhammer symbol defined by

$$
(\alpha)_{0}=1, \quad(\alpha)_{k}=\alpha(\alpha+1) \ldots(\alpha+k-1) ; k \in \mathrm{N} .
$$

Then using the identity ([5]):

$$
z\left(H_{s}^{q}\left[\alpha_{i}\right] f\right)^{\prime}(z)=\alpha_{i}\left(H_{s}^{q}\left[\alpha_{i}+1\right] f(z)\right)-\left(\alpha_{i}-p\right)\left(H_{s}^{q}\left[\alpha_{i}\right] f(z)\right)
$$

$(i=1, \ldots, q)$ in $(1.5)$, we see that the class $\mathscr{J}_{p}^{m}(g ; \alpha, A, B)$ reduces to a known function class studied by Liu [8].

It may be observed here that the linear operator

$$
\left(H_{s}^{q}\left[\alpha_{1}\right] f\right)(z):=H_{s}^{q}\left(\alpha_{1}, \ldots, \alpha_{q} ; \beta_{1}, \ldots, \beta_{s}\right) f(z)
$$

involved in the identity (1.9) is the Dziok-Srivastava linear operator [5] (see also [6]) used in many of the recent works, and includes such well known operators as the Hohlov linear operator, Saitoh generalized linear operator, the Carlson-Shaffer linear operator, the Ruscheweyh derivative operator, the Bernardi-Libera-Livingston operator, and the Srivastave-Owa fractional derivative operator (as well as their various generalized versions). One may refer to the papers [5] and [6] for further details and references for these operators. The Dziok- Srivastava linear operator introduced in [5] has further been generalized by Dziok and Raina [3] (see also [4]). A recent class of analytic functions due to Aouf and Darwish [2] is also obtainable from our class defined by (1.5) by suitably specializing the parameters in (1.5) and the sequence $b_{k}$ in (1.2) by taking advantage of (1.8). Furthermore, for $m=0$, the class $\mathscr{J}_{p}^{0}(g ; \alpha, A, B)$ was investigated very recently in [12] in which different characteristics and properties from those presented in this paper were studied, and one may refer to this paper for additional references to the works which exhibit relevant connections with other known function classes.

The present paper derives various useful properties and characteristics of the function classes $\mathscr{J}_{p}^{m}(g ; \alpha, A, B), \mathscr{J}_{p}^{*}(g ; \alpha, \beta, m)$ and their associated classes (defined above) by using differential subordination methods. Various consequences of the main results lead to certain corollaries whose connections (and particular cases) with known and new results are briefly mentioned. 


\section{Prelimininaries and key lemmas}

In the sequel, we require the following lemmas to investigate the function classes $\mathscr{J}_{p}^{m}(g ; \alpha, A, B), \mathscr{J}_{p}^{*}(g ; \alpha, \beta, m)$ and their associated classes (defined above).

Lemma 1. A function $f \in \mathscr{A}_{p}$ is in the class $\mathrm{J}_{p}^{0}(g ; \alpha, A, B)$ if and only if

$$
\left|(1-\alpha) \frac{(f * g)(z)}{z^{p}}+\frac{\alpha}{p} \frac{(f * g)^{\prime}(z)}{z^{p-1}}-\frac{1-A B}{1-B^{2}}\right|<\frac{A-B}{1-B^{2}}
$$

$(-1 \leq B<A \leq 1 ; z \in \mathrm{U})$.

The proof of Lemma 1 is similar to that of a known result [7, Lemma 1]. The details are hence omitted.

Lemma 2 (Miller and Mocanu [10]). Let $h(z)$ be a convex (univalent) function in $\mathrm{U}$ with $h(0)=1$, and let the function $\phi(z)=1+p_{1} z+p_{2} z^{2}+\cdots$ be analytic in $\mathrm{U}$. If

$$
\phi(z)+\frac{z \phi^{\prime}(z)}{\gamma} \prec h(z)
$$

for $\gamma \neq 0$ and $\Re(\gamma) \geq 0$, then

$$
\phi(z) \prec \psi(z):=\frac{\gamma}{z^{\gamma}} \int_{0}^{z} t^{\gamma-1} h(t) d t \prec h(z)
$$

and $\psi(z)$ is the best dominant.

Lemma 3 (see [11, Theorem 8]). Let a function $f(z)$ of the form (1.1) be analytic in $\mathrm{U}$. If there exists $a(p-m+1)$-valent starlike function of the form

$$
g(z)=z^{p-m+1}+\sum_{k=p-m+2}^{\infty} a_{k} z^{k}
$$

in $\mathrm{U}$ such that

$$
\Re\left(\frac{z f^{(m)}(z)}{g(z)}\right)>0 \quad(z \in \mathrm{U}),
$$

then $f(z)$ is p-valent in $\mathrm{U}$.

The generalized hypergeometric function ${ }_{q} F_{s}$ is defined by (cf., e.g. [5])

$$
{ }_{q} F_{s}(z) \equiv{ }_{q} F_{s}\left(\alpha_{1}, \ldots, \alpha_{q} ; \beta_{1}, \ldots, \beta_{s} ; z\right)=: \sum_{n=0}^{\infty} \frac{\left(\alpha_{1}\right)_{n} \ldots\left(\alpha_{q}\right)_{n}}{\left(\beta_{1}\right)_{n} \ldots\left(\beta_{s}\right)_{n}} \cdot \frac{z^{n}}{n !}
$$


$\left(z \in \mathrm{U} ; \alpha_{j} \in \mathrm{C}(j=1, \ldots, q), \beta_{j} \in \mathrm{C} \backslash\{0,-1,-2, \ldots\}(j=1, \ldots, s), q \leq\right.$ $\left.s+1 ; q, s \in \mathrm{N}_{0}\right)$.

The following identities to be used in establishing our results are fairly well known ([1, pp. 556-558]).

Lemma 4. For real or complex numbers $a, b$ and $c(c \neq 0,-1,-2, \ldots)$ :

$$
\int_{0}^{1} t^{b-1}(1-t)^{c-b-1}(1-z t)^{-a} d t=\frac{\Gamma(a) \Gamma(c-b)}{\Gamma(c)}{ }_{2} F_{1}(a, b ; c ; z),
$$

$$
F_{1}(a, b ; c ; z)=(1-z)_{2}^{-a} F_{1}\left(a, c-b ; c ; \frac{z}{z-1}\right)
$$

$$
{ }_{2} F_{1}\left(1,1 ; 3 ; \frac{z}{z-1}\right)=\frac{2(z-1)}{z}\left[1+\frac{\ln (1-z)}{z}\right] .
$$

For a function $f \in \mathscr{A}_{p}$ given by (1.1), the generalized Bernardi-LiberaLivingston integral operator (c.f. [13]) is defined by

$$
\begin{aligned}
\mathscr{K}_{p, \gamma}(f)(z) & :=\frac{\gamma+p}{z^{\gamma}} \int_{0}^{z} t^{\gamma-1} f(t) d t \\
& =z^{p}+\sum_{k=p+1}^{\infty} \frac{\gamma+p}{\gamma+k} a_{k} z^{k} \quad(\gamma>-p ; p \in \mathbf{N}) .
\end{aligned}
$$

It readily follows from (2.7) that the operator $\mathscr{K}_{p, \gamma}(f)(z)$ is a self-preserving operator on $\mathscr{A}_{p}$, so that

$$
f(z) \in \mathscr{A}_{p} \Rightarrow \mathscr{K}_{p, \gamma}(f)(z) \in \mathscr{A}_{p} \quad(\gamma>-p ; p \in \mathrm{N}) .
$$

\section{Main results and their consequences}

Our first main result is contained in the following:

Theorem 1. Let $\alpha>0, p>m\left(m \in \mathrm{N}_{0}\right), A \in \mathrm{C}, B \in[-1,0], A \neq B$. If $f(z) \in \mathscr{J}_{p}^{m}(g ; \alpha, A, B)$, then

$$
\frac{r(p, m)(f * g)^{(m)}(z)}{z^{p-m}} \prec \mathscr{X}(z) \prec \frac{1+A z}{1+B z} \quad(z \in \mathrm{U}),
$$

where

$$
\mathscr{X}(z)= \begin{cases}\frac{A}{B}+\left(1-\frac{A}{B}\right)(1+B z)^{-1} F_{1}\left(1,1 ; \frac{p-m}{\alpha}+1 ; \frac{B z}{B z+1}\right) & (B \neq 0) ; \\ 1+\frac{p-m}{\alpha+p-m} A z & (B=0),\end{cases}
$$


and $\mathscr{X}(z)$ is the best dominant of (3.1). Also,

$$
\Re\left(\frac{r(p, m)(f * g)^{(m)}(z)}{z^{p-m}}\right)>\left.\mathscr{X}(-1)\right|_{A=\Re(A)} .
$$

The result (3.2) is sharp.

Proof. Suppose $f(z) \in \mathscr{J}_{p}^{m}(g ; \alpha, A, B)$, and assume that

$$
\frac{r(p, m)(f * g)^{(m)}(z)}{z^{p-m}}=\theta(z) .
$$

We observe that the function $\theta(z)$ is of the form

$$
\theta(z)=1+c_{1} z+c_{2} z^{2}+\cdots,
$$

which is analytic in $U$ with $\theta(0)=1$. Now differentiating (3.3) with respect to $z$, we get

$$
\frac{r(p, m+1)(f * g)^{(m+1)}(z)}{z^{p-m-1}}=\theta(z)+\frac{1}{p-m} z \theta^{\prime}(z)
$$

and applying (1.5), (3.3) and (3.4), we arrive at

$$
\begin{aligned}
(1-\alpha) \frac{r(p, m)(f * g)^{(m)}(z)}{z^{p-m}} & +\alpha \frac{r(p, m+1)(f * g)^{(m+1)}(z)}{z^{p-m-1}} \\
& =\theta(z)+\frac{\alpha}{p-m} z \theta^{\prime}(z) \prec \frac{1+A z}{1+B z} \quad(z \in \mathbf{U}) .
\end{aligned}
$$

The result (3.1) follows on using Lemma 2. Also, in view of Lemma 4, we observe that

$$
\begin{aligned}
\theta(z) & \prec \frac{p-m}{\alpha} z^{-\frac{(p-m)}{\alpha}} \int_{0}^{z} t^{\frac{p-m}{\alpha}-1} \frac{1+A t}{1+B t} d t \\
& = \begin{cases}\frac{A}{B}+\left(1-\frac{A}{B}\right)(1+B z)^{-1}{ }_{2} F_{1}\left(1,1 ; \frac{p-m}{\alpha}+1 ; \frac{B z}{B z+1}\right) & (B \neq 0) \\
1+\frac{p-m}{\alpha+p-m} A z & (B=0)\end{cases} \\
& =\mathscr{X}(z) .
\end{aligned}
$$

To establish (3.2), we infer (under the conditions stated with Theorem 1) 
that

$$
\begin{aligned}
\Re\left(\frac{r(p, m)(f * g)^{(m)}(z)}{z^{p-m}}\right) & =\frac{p-m}{\alpha} \int_{0}^{1} u^{\frac{p-m}{\alpha}-1} \Re\left(\frac{1+A u w(z)}{1+B u w(z)}\right) d u \\
& >\frac{p-m}{\alpha} \int_{0}^{1} u^{\frac{p-m}{\alpha}-1} \frac{1-\Re(A) u}{1-B u} d u .
\end{aligned}
$$

The sharpness of the result (3.2) can be established by considering the functions $\mathscr{X}(z)$ defined by (3.5). It is sufficient to show that

$$
\inf _{|z|<1}\left\{\mathfrak{R}(\mathscr{X}(z)\}=\left.\mathscr{X}(-1)\right|_{A=\Re(A)} .\right.
$$

We observe from (3.5) that (for $|z| \leq r(0<r<1)$ )

$$
\begin{aligned}
\Re\{\mathscr{X}(z)\} & \geq \frac{p-m}{\alpha} \int_{0}^{1} u^{\frac{p-m}{\alpha}-1} \mathfrak{R}\left(\frac{1+A u r}{1+B u r}\right) d u \\
& =\left.\left.\mathscr{X}(-r)\right|_{A=\Re(A)} \rightarrow \mathscr{X}(-1)\right|_{A=\Re(A)} \text { as } r \rightarrow 1-,
\end{aligned}
$$

and this completes the proof of Theorem 1 .

If we use the elementary inequality

$$
\Re\left(w^{\frac{1}{v}}\right) \geq(\Re(w))^{\frac{1}{v}} \quad \text { for } \quad \Re(w)>0 \text { and } v \geq 1,
$$

then Theorem 1 gives

Corollary 1. If $f(z) \in \mathscr{J}_{p}^{m}(g ; \alpha, A, B)$, then

$$
\begin{aligned}
& \Re\left[\left(\frac{r(p, m)(f * g)^{(m)}(z)}{z^{p-m}}\right)^{\frac{1}{v}}\right] \\
> & {\left[\frac{\Re(A)}{B}+\left(1-\frac{\Re(A)}{B}\right)(1-B)^{-1}{ }_{2} F_{1}\left(1,1 ; \frac{p-m}{\alpha}+1 ; \frac{B}{B-1}\right)\right]^{\frac{1}{v}} }
\end{aligned}
$$

$(B \neq 0 ; v \geq 1)$.

REMARK 1. We observe that if we use the parametric substitutions given by (1.8) and apply the identity (1.9), then Theorem 1 and Corollary 1 would yield the results given recently by Liu [8, p. 3, Theorem 2.4]. Another known result due to Srivastava et al. [13, p. 5, Theorem 1] is easily obtainable from Theorem 1 when the coefficients $b_{k}$ in (1.2) and the parameters in (1.5) are chosen as follows:

$$
b_{k}=\left(\frac{k+1}{p+1}\right)^{r} \quad\left(p \in \mathrm{N} ; r \in \mathrm{N}_{0}\right), \quad m=0 \quad \text { and } \quad \alpha=1 .
$$


Upon setting

$$
A=1-\frac{2 \beta}{p-m} \quad(0 \leq \beta<p, p>m) \quad \text { and } \quad B=-1
$$

in Theorem 1 and using (1.6), we get

Corollary 2. If $f(z) \in \mathscr{J}_{p}^{*}(g ; \alpha, \beta, m)$, then

(3.8) $\Re\left(\frac{r(p, m)(f * g)^{(m)}(z)}{z^{p-m}}\right)$

$$
>\frac{\beta}{p-m}+\left(1-\frac{\beta}{p-m}\right)\left[{ }_{2} F_{1}\left(1,1 ; \frac{p-m}{\alpha}+1 ; \frac{1}{2}\right)-1\right] .
$$

The result is the best possible.

Theorem 2. Let $f(z) \in \mathrm{J}_{p}^{m}\left(g ; \alpha, A^{*}, B\right)\left(\alpha>0, p>m,-1 \leq B<A^{*} \leq\right.$ $1 ; B \neq 0 ; z \in \mathrm{U})$, then

$$
\Re\left(\frac{r(p, m)(f * g)^{(m)}(z)}{z^{p-m}}\right)>0 \quad(z \in \mathrm{U}),
$$

where $A^{*}$ is given by

$$
A^{*}=\frac{B_{2} F_{1}\left(1,1, \frac{p-m}{\alpha}+1 ; \frac{B}{B-1}\right)}{{ }_{2} F_{1}\left(1,1 ; \frac{p-m}{\alpha}+1 ; \frac{B}{B-1}\right)+(B-1)} .
$$

The result (3.9) is sharp.

Proof. In view of Theorem 1, if

$$
\begin{aligned}
(1-\alpha) \frac{r(p, m)(f * g)^{(m)}(z)}{z^{p-m}} & \\
+\alpha \frac{r(p, m+1)(f * g)^{(m+1)}(z)}{z^{p-m-1}} & \prec \frac{1+A^{*} z}{1+B z} \quad(z \in \mathrm{U}),
\end{aligned}
$$

then

(3.12) $\Re\left(\frac{r(p, m)(f * g)^{(m)}(z)}{z^{p-m}}\right)$

$$
>\frac{A^{*}}{B}+\left(1-\frac{A^{*}}{B}\right)(1-B)^{-1} F_{1}\left(1,1 ; \frac{p-m}{\alpha}+1 ; \frac{B}{B-1}\right) .
$$


On substituting the value of $A^{*}$ given by (3.10) in the right-hand side of the above inequality (3.12), we readily get

$$
\Re\left(\frac{r(p, m)(f * g)^{(m)}(z)}{z^{p-m}}\right)>0 \quad(z \in \mathrm{U}),
$$

which proves Theorem 2 .

Putting

$$
m=0, \quad \alpha=\frac{p}{p+1}
$$

and

$$
g(z)=z^{p}+\sum_{k=p+1}^{\infty} \frac{k}{p} z^{k}=z^{p}\left\{1+\frac{(p+1) z}{p}{ }_{2} F_{1}(1, p+2 ; p+1 ; z)\right\}
$$

in Theorem 2, we get (in view of Lemma 3) the following:

Corollary 3. If $f(z) \in \mathscr{A}_{p}$ such that

$$
\frac{2 f^{\prime}(z)+z f^{\prime \prime}(z)}{p(p+1) z^{p-1}} \prec \frac{1+A_{1} z}{1+B z} \quad\left(-1 \leq B<A_{1} \leq 1, B \neq 0 ; z \in \mathrm{U}\right),
$$

where $A_{1}$ is given by

$$
A_{1}=\frac{B_{2} F_{1}\left(1,1 ; p+2 ; \frac{B}{B-1}\right)}{{ }_{2} F_{1}\left(1,1 ; p+2 ; \frac{B}{B-1}\right)+(B-1)},
$$

then

$$
\Re\left(\frac{f^{\prime}(z)}{z^{p-1}}\right)>0 \quad(z \in U)
$$

and $f(z)$ is hence p-valent in $\mathrm{U}$.

REMARK 2. (i) Corollary 3 provides the corrected form of a known result due to Srivastava et al. [13, p. 6, Corollary 2].

(ii) For $p=1$, we note that Corollary 3 in view of (2.6) yields an assertion which we express as follows:

If

$$
f^{\prime}(z)+\frac{1}{2} f^{\prime \prime}(z) \prec \frac{1+A_{2} z}{1+B z}
$$

$\left(f \in \mathscr{A}\left(=\mathscr{A}_{1}\right),-1 \leq B<A_{2} \leq 1, B \neq 0 ; z \in \mathrm{U}\right)$, where $A_{2}$ is given by

$$
A_{2}=\frac{2 B[B+\ln (1-B)]}{2[B+\ln (1-B)]+B^{2}},
$$


then

$$
\Re\left(f^{\prime}(z)\right)>0 \quad(z \in U),
$$

and hence $f(z)$ is univalent in $\mathrm{U}$. Further, on choosing $B=-1$ in (3.13) and using the principal of subordination, we arrive at the following assertion:

If

$$
\Re\left(f^{\prime}(z)+\frac{1}{2} f^{\prime \prime}(z)\right)>\frac{4 \ln 2-3}{4 \ln 2-2}=-0.2943 \quad(f \in \mathscr{A} ; z \in \mathrm{U})
$$

then

$$
\Re\left(f^{\prime}(z)\right)>0 \quad(z \in \mathrm{U}) .
$$

THEOREM 3. If $f \in \mathscr{A}_{p}$ satisfies the following inequality:

$$
\mathfrak{R}\left(\frac{r(p, m)(f * g)^{(m)}(z)}{z^{p-m}}\right)>\frac{\beta}{p-m}
$$

$(0 \leq \beta<p, p>m ; z \in \mathrm{U})$, then

$$
\begin{aligned}
\mathfrak{R}\left((1-\alpha) \frac{r(p, m)(f * g)^{(m)}(z)}{z^{p-m}}\right. & \\
\left.+\alpha \frac{r(p, m+1)(f * g)^{(m+1)}(z)}{z^{p-m-1}}\right)>\frac{\beta}{p-m} & \left(|z|<R_{1}\right),
\end{aligned}
$$

where $R_{1}$ is given by

$$
R_{1}:=\frac{\sqrt{\alpha^{2}+(p-m)^{2}}-\alpha}{p-m} \quad(p \in \mathrm{N} ; \alpha>0, p>m) .
$$

The result is sharp.

Proof. Let $f(z) \in \mathscr{A}_{p}$ satisfy the inequality (3.14) and suppose that

$$
\frac{r(p, m)(f * g)^{(m)}(z)}{z^{p-m}}=\frac{\beta}{p-m}+\left(1-\frac{\beta}{p-m}\right) w(z),
$$

where

$$
w(z)=1+a_{1} z+a_{2} z^{2}+\cdots
$$

is analytic and has positive real part in U. Differentiating (3.16) with respect to $z$, we get

$$
\begin{aligned}
& \frac{r(p, m+1)(f * g)^{(m+1)}(z)}{z^{p-m-1}} \\
& \quad=\frac{\beta}{p-m}+\left(1-\frac{\beta}{p-m}\right) w(z)+\frac{1}{p-m}\left(1-\frac{\beta}{p-m}\right) z w^{\prime}(z),
\end{aligned}
$$


which in view of (1.7) yields

$$
\begin{aligned}
& (1-\alpha) \frac{r(p, m)(f * g)^{(m)}(z)}{z^{p-m}}+\alpha \frac{r(p, m+1)(f * g)^{(m+1)}(z)}{z^{p-m-1}} \\
& -\frac{\beta}{p-m}=\left(1-\frac{\beta}{p-m}\right)\left[w(z)+\frac{\alpha}{p-m} z w^{\prime}(z)\right]
\end{aligned}
$$

Applying the well-known estimate (cf., e.g. [13]):

$$
\frac{\left|z w^{\prime}(z)\right|}{\Re\{w(z)\}} \leq \frac{2 r}{1-r^{2}} \quad(|z|=r<1)
$$

in (3.17), we deduce that

$$
\begin{array}{r}
\Re\left((1-\alpha) \frac{r(p, m)(f * g)^{(m)}(z)}{z^{p-m}}+\alpha \frac{r(p, m+1)(f * g)^{(m+1)}(z)}{z^{p-m-1}}-\frac{\beta}{p-m}\right) \\
\geq\left(1-\frac{\beta}{p-m}\right) \Re\{w(z)\}\left(1-\frac{2 \alpha r}{(p-m)\left(1-r^{2}\right)}\right) .
\end{array}
$$

It follows easily that the right-hand side of (3.19) is positive when $|w(z)|<R_{1}$ (where $R_{1}$ is given by (3.15)), which implies that $f(z) \in \mathscr{J}_{p}^{*}(g ; \alpha, \beta, m)$ for $|z|<R_{1}$.

To show that the bound $R_{1}$ is sharp, we consider the function $f \in \mathscr{A}_{p}$ defined by

$$
\frac{r(p, m)(f * g)^{(m)}(z)}{z^{p-m}}=\frac{\beta}{p-m}+\left(1-\frac{\beta}{p-m}\right) \frac{1+z}{1-z} \quad(z \in \mathrm{U}) .
$$

Evidently

$$
\begin{aligned}
(1-\alpha) \frac{r(p, m)(f * g)^{(m)}(z)}{z^{p-m}} & +\alpha \frac{r(p, m+1)(f * g)^{(m+1)}(z)}{z^{p-m-1}}-\frac{\beta}{p-m} \\
= & \left(1-\frac{\beta}{p-m}\right)\left[\frac{(p-m)\left(1-z^{2}\right)+2 \alpha z}{(1-z)^{2}}\right]=0
\end{aligned}
$$

for $z=-R_{1}$, which completes the proof of Theorem 3 .

For

$$
m=0, \quad \alpha=\frac{p}{p+1} \quad \text { and } \quad g(z)=\frac{z^{p}}{1-z},
$$

Theorem 3 gives 
Corollary 4. If $f \in \mathscr{A}_{p}$ satisfies the following inequality:

$$
\Re\left(\frac{f(z)}{z^{p}}\right)>\frac{\beta}{p} \quad(0 \leq \beta<p: z \in \mathrm{U})
$$

then

$$
\Re\left(\frac{f(z)+z f^{\prime}(z)}{z^{p}}\right)>\beta\left(1+\frac{1}{p}\right) \quad\left(|z|<\frac{\sqrt{(p+1)^{2}+1}-1}{p+1}\right) .
$$

The result is sharp.

Remark 3. We observe from Corollary 4 that if $f \in \mathscr{A}$ satisfies the following inequality:

$$
\Re\left(\frac{f(z)}{z}\right)>\beta \quad(0 \leq \beta<1 ; z \in \mathrm{U}),
$$

then

$$
\Re\left(\frac{f(z)}{z}+f^{\prime}(z)\right)>2 \beta \quad\left(|z|<\frac{\sqrt{5}-1}{2}\right) .
$$

The result is sharp.

TheOREM 4. If $f(z) \in \mathscr{J}_{p}^{m}(g ; \alpha, A, B)$, then

$$
\begin{aligned}
(1-\alpha) \frac{r(p, m)\left(\left(\mathscr{K}_{p, \gamma} f\right) * g\right)^{(m)}(z)}{z^{p-m}} & \\
& +\alpha \frac{r(p, m+1)\left(\left(\mathscr{K}_{p, \gamma} f\right) * g\right)^{(m+1)}(z)}{z^{p-m-1}} \prec \tau(z) \prec \frac{1+A z}{1+B z}
\end{aligned}
$$

$\left(\alpha>0, p>m\left(m \in \mathrm{N}_{0}\right), A \in \mathrm{C}, B \in[-1,0], A \neq B ; z \in \mathrm{U}\right)$, where $\mathscr{K}_{p, \gamma}$ is defined by (2.7) and the function $\tau(z)$ is given by

$$
\tau(z)= \begin{cases}\frac{A}{B}+\left(1-\frac{A}{B}\right)(1+B z)_{2}^{-1} F_{1}\left(1,1 ; \gamma+p+1 ; \frac{B z}{B z+1}\right) & (B \neq 0) ; \\ 1+\frac{\gamma+p}{\gamma+p+1} A z & (B=0),\end{cases}
$$

is the best dominant of (3.20). Furthermore

$$
\begin{aligned}
& \mathfrak{R}\left((1-\alpha) \frac{r(p, m)\left(\left(\mathscr{K}_{p, \gamma} f\right) * g\right)^{(m)}(z)}{z^{p-m}}\right. \\
& \left.\quad+\alpha \frac{r(p, m+1)\left(\left(\mathscr{K}_{p, \gamma} f\right) * g\right)^{(m+1)}(z)}{z^{p-m-1}}\right)>\left.\tau(-1)\right|_{A=\Re(A)}
\end{aligned}
$$


The result (3.21) is the best possible.

Proof. Let $f(z) \in \mathscr{J}_{p}^{m}(g ; \alpha, A, B)$, and assume that

$$
\begin{aligned}
& (1-\alpha) \frac{r(p, m)\left(\left(\mathscr{K}_{p, \gamma} f\right) * g\right)^{(m)}(z)}{z^{p-m}} \\
& \quad+\alpha \frac{r(p, m+1)\left(\left(\mathscr{K}_{p, \gamma} f\right) * g\right)^{(m+1)}(z)}{z^{p-m-1}}=q(z),
\end{aligned}
$$

where

$$
q(z)=1+d_{1} z+d_{2} z^{2}+\cdots
$$

is analytic in $\mathrm{U}$ with $q(0)=1$. Using the identity that

$$
\begin{aligned}
z\left(\left(\mathscr{K}_{p, \gamma} f\right) * g\right)^{(m+1)}(z) & \\
& =(\gamma+p)(f * g)^{(m)}(z)-(\gamma+m)\left(\left(\mathscr{K}_{p, \gamma} f\right) * g\right)^{(m)}(z)
\end{aligned}
$$

in (3.22), and differentiating the resulting equation with respect to $z$, we obtain

$$
\begin{aligned}
(1-\alpha) \frac{r(p, m)(f * g)^{(m)}(z)}{z^{p-m}}+\alpha & \frac{r(p, m+1)(f * g)^{(m+1)}(z)}{z^{p-m-1}} \\
& =q(z)+\frac{z q^{\prime}(z)}{\gamma+p} \prec \frac{1+A z}{1+B z} \quad(z \in \mathrm{U}),
\end{aligned}
$$

which on applying Lemma 2 (when $\gamma$ is replaced by $\gamma+p$ ) yields

$$
q(z) \prec(\gamma+p) z^{-(\gamma+p)} \int_{0}^{z} t^{(\gamma+p)-1} \frac{1+A t}{1+B t} d t .
$$

The assertion (3.20) and the estimate (3.21) can now be deduced on the same lines as given in the proof of Theorem 1 . This evidently finishes the proof of Theorem 4.

Putting

$$
A=1-\frac{2 \beta}{p} \quad(0 \leq \beta<p) \quad \text { and } \quad B=-1
$$

in Theorem 4 and using (1.6), we get

Corollary 5. If $f(z) \in \mathscr{J}_{p}^{*}(g ; \alpha, \beta, 0)$, then the function $\mathscr{K}_{p, \gamma} f$ defined by (2.7) belongs to the class $\mathscr{J}_{p}^{*}(g ; \alpha, \psi(p, \beta, \gamma), 0)$, where

$$
\psi(p, \beta, \gamma)=\beta+(p-\beta)\left[{ }_{2} F_{1}\left(1,1 ; p+\gamma+1 ; \frac{1}{2}\right)-1\right] .
$$

The result is the best possible. 
THeOREM 5. If $\mathscr{K}_{p, \gamma} f(z) \in \mathscr{J}_{p}^{*}(g ; \alpha, \beta, m)$, then

$\Re\left((1-\alpha) \frac{r(p, m)(f * g)^{(m)}(z)}{z^{p-m}}+\alpha \frac{r(p, m+1)(f * g)^{(m+1)}(z)}{z^{p-m-1}}\right)>\frac{\beta}{p-m}$

in $|z|<R_{2}$, where $R_{2}$ is given by

$$
R_{2}:=\frac{\sqrt{(\gamma+p)^{2}+1}-1}{\gamma+p} \quad(\gamma>-p)
$$

The bound $R_{2}$ is sharp.

Proof. Since $f(z) \in \mathscr{J}_{p}^{*}(g ; \alpha, \beta, m)$, let us suppose that

$$
\begin{aligned}
& (1-\alpha) \frac{r(p, m)\left(\left(\mathscr{K}_{p, \gamma} f\right) * g\right)^{(m)}(z)}{z^{p-m}} \\
+\alpha & \frac{r(p, m+1)\left(\left(\mathscr{K}_{p, \gamma} f\right) * g\right)^{(m+1)}(z)}{z^{p-1}}=\frac{\beta}{p-m}+\left(1-\frac{\beta}{p-m}\right) w(z),
\end{aligned}
$$

where $w(z)$ is analytic in $\mathrm{U}$ with

$$
w(0)=1 \quad \text { and } \quad \Re(w(z))>0 .
$$

Using (3.23) in (3.25) and differentiating the resulting equation with respect to $z$, we obtain

$$
\begin{aligned}
& \Re\left((1-\alpha) \frac{r(p, m)(f * g)^{(m)}(z)}{z^{p-m}}+\alpha \frac{r(p, m+1)(f * g)^{(m+1)}(z)}{z^{p-m-1}}\right) \\
&=\frac{\beta}{p-m}+\left(1-\frac{\beta}{p-m}\right) \Re\left(w(z)+\frac{z w^{\prime}(z)}{\gamma+p}\right) .
\end{aligned}
$$

Applying (3.18) in (3.26), and following the same lines of proof of Theorem 3, we arrive at the assertion of Theorem 5 .

Lastly, we prove the following result concerning the class $\mathrm{J}_{p}^{0}(g ; \alpha, A, B)$.

THEOREM 6. If each of the functions $f(z)$ given by (1.1) and

$$
h(z)=z^{p}+\sum_{k=p+1}^{\infty} c_{k} z^{k}
$$

belongs to the class $\mathrm{J}_{p}^{0}(g ; \alpha, A, B)$, then so does the function $(f * h)(z)$. 
Proof. By virtue of Lemma 1, we need to show that

$$
\left|(1-\alpha) \frac{[(f * h) * g](z)}{z^{p}}+\frac{\alpha}{p} \frac{[(f * h) * g]^{\prime}(z)}{z^{p-1}}-\xi\right|<\eta \quad(z \in \mathrm{U}),
$$

where

$$
\xi=\frac{1-A B}{1-B^{2}} \quad \text { and } \quad \eta=\frac{A-B}{1-B^{2}} .
$$

It is known from the Gutzmer inequality (cf., e.g. [9, p. 359]) that if $F(z)=\sum_{k=0}^{\infty} w_{k} z^{k}$ is analytic in $\mathrm{U}$ and $|F(z)|<M$, then

$$
\sum_{k=0}^{\infty}\left|w_{k}\right|^{2}<M^{2}
$$

Since by hypothesis $f(z) \in \mathrm{J}_{p}^{0}(g ; \alpha, A, B)$, and in view of Lemma 1 and the above inequality (3.28), we get

$$
(1-\xi)^{2}+\sum_{k=p+1}^{\infty}\left[\frac{\alpha(k-p)+p}{p} b_{k}\right]^{2}\left|a_{k}\right|^{2}<\left(\frac{A-B}{1-B^{2}}\right)^{2},
$$

which implies that

$$
\sum_{k=p+1}^{\infty}\left[\frac{\alpha(k-p)+p}{p} b_{k}\right]^{2}\left|a_{k}\right|^{2}<\frac{(A-B)^{2}}{1-B^{2}} .
$$

Similarly, we can show that

$$
\sum_{k=p+1}^{\infty}\left[\frac{\alpha(k-p)+p}{p} b_{k}\right]^{2}\left|c_{k}\right|^{2}<\frac{(A-B)^{2}}{1-B^{2}} .
$$

Applying the Cauchy-Schwarz inequality for $|z|=r<1$, we find that

$$
\begin{aligned}
\mid(1- & \alpha) \frac{[(f * h) * g](z)}{z^{p}}+\frac{\alpha}{p} \frac{[(f * h) * g]^{\prime}(z)}{z^{p-1}}-\left.\xi\right|^{2} \\
& =\left|(1-\xi)+\sum_{k=p+1}^{\infty}\left[\frac{\alpha(k-p)+p}{p} b_{k}\right]^{2} a_{k} c_{k} z^{k-p}\right|^{2} \\
& \leq(1-\xi)^{2}+2(1-\xi) \sum_{k=p+1}^{\infty}\left[\frac{\alpha(k-p)+p}{p} b_{k}\right]^{2}\left|a_{k}\right|\left|c_{k}\right| r^{k-p}
\end{aligned}
$$




$$
\begin{aligned}
& +\left|\sum_{k=p+1}^{\infty}\left[\frac{\alpha(k-p)+p}{p} b_{k}\right]^{2}\right| a_{k}|| c_{k}\left|r^{k-p}\right|^{2} \\
& \leq(1-\xi)^{2}+2(1-\xi)\left[\sum_{k=p+1}^{\infty}\left[\frac{\alpha(k-p)+p}{p} b_{k}\right]^{2}\left|a_{k}\right|^{2} r^{k-p}\right]^{\frac{1}{2}} \\
& \cdot\left[\sum_{k=p+1}^{\infty}\left[\frac{\alpha(k-p)+p}{p} b_{k}\right]^{2}\left|c_{k}\right|^{2} r^{k-p}\right]^{\frac{1}{2}} \\
& +\left[\sum_{k=p+1}^{\infty}\left[\frac{\alpha(k-p)+p}{p} b_{k}\right]^{2}\left|a_{k}\right|^{2} r^{k-p}\right] \\
& \cdot\left[\sum_{k=p+1}^{\infty}\left[\frac{\alpha(k-p)+p}{p} b_{k}\right]^{2}\left|a_{k}\right|^{2} r^{k-p}\right] \\
& \leq(1-\xi)^{2}+2(1-\xi)\left[\sum_{k=p+1}^{\infty}\left[\frac{\alpha(k-p)+p}{p} b_{k}\right]^{2}\left|a_{k}\right|^{2}\right]^{\frac{1}{2}} \\
& \cdot\left[\sum_{k=p+1}^{\infty}\left[\frac{\alpha(k-p)+p}{p} b_{k}\right]^{2}\left|c_{k}\right|^{2}\right]^{\frac{1}{2}} \\
& +\left[\sum_{k=p+1}^{\infty}\left[\frac{\alpha(k-p)+p}{p} b_{k}\right]^{2}\left|a_{k}\right|^{2}\right] \\
& \cdot\left[\sum_{k=p+1}^{\infty}\left[\frac{\alpha(k-p)+p}{p} b_{k}\right]^{2}\left|a_{k}\right|^{2}\right] \\
& <(1-\xi)^{2}+2(1-\xi) \frac{(A-B)^{2}}{1-B^{2}}+\frac{(A-B)^{4}}{\left(1-B^{2}\right)^{2}} \\
& =(1-\xi)^{2}+\frac{2 B(A-B)^{3}}{\left(1-B^{2}\right)^{2}}+\frac{(A-B)^{4}}{\left(1-B^{2}\right)^{2}} \\
& =\frac{A^{2}(A-B)^{2}}{\left(1-B^{2}\right)^{2}}<\eta^{2} \text {, }
\end{aligned}
$$

on using (3.29) and (3.30). By virtue of Lemma 1, we infer that $(f * h)(z) \in$ $\mathrm{J}_{p}^{0}(g ; \alpha, A, B)$.

REMARK 4. On setting

$$
\alpha=\frac{1}{p} \quad \text { and } \quad b_{k}=\frac{k}{p}\left(\frac{p+k}{p+1}\right)^{\delta}
$$


in Theorem 6, we obtain a known result due to Srivastava et al. [13, p. 12, Theorem 10].

Acknowledgement. The authors express their sincerest thanks to Professor Robert Magnus for suggestions.

\section{REFERENCES}

1. Abramowitz, M., and Stegun, I. A. (eds.), Handbook of Mathematical Functions with Formulas, Graphs and Mathematical Tables, Dover, New York 1971.

2. Aouf, M. K., and Darwish, H. E., Subclasses of analytic functions associated with the generalized hypergeometric function, Comput. Math. Appl. 57 (2009), 356-366.

3. Dziok, J., and Raina, R. K., Families of analytic functions associated with the Wright generalized hypergeometric function, Demonstratio Math. 37 (2004), 533-542.

4. Dziok, J., Raina, R. K., and Srivastava, H. M., Some classes of analytic functions associated with operators on Hilbert space involving Wright's generalized hypergeometric functions, Proc. Jangjeon Math. Soc. 7 (2004), 43-55.

5. Dziok, J., and Srivastava, H. M., Classes of analytic functions associated with the generalized hypergeometric function, Appl. Math. Comput. 103 (1999), 1-13.

6. Dziok, J., and Srivastava, H. M., Certain subclasses of analytic functions associated with the generalized hypergeometric function, Integral Transforms Spec. Funct. 14 (2003), 7-18.

7. Janowski, W., Some extremal problems for certain families of analytic functions I, Ann. Polon. Math. 28 (1973), 297-326.

8. Liu, J.-L., On subordination for certain multivalent analytic functions associated with the generalized hypergeometric function, J. Inequal. Pure Appl. Math. 7 (2006), Article 131.

9. Markushevich, A. I., Theory of Functions of a Complex Variable, Vol. 1, Chelsea, New York 1985.

10. Miller, S. S., and Mocanu, P. T., Differential subordinations and univalent functions, Michigan Math. J. 28 (1981), 157-171.

11. Nunokawa, M., On the theory of multivalent functions, Tsukuba J. Math. 11 (1987), 273-286.

12. Prajapat, J. K., and Raina, R. K., Certain results on differential subordination for some classes of multivalently analytic functions associated with a convolution structure, Bull. Belg. Math. Soc. Simon Stevin 16 (2009), 153-165.

13. Srivastava, H. M., Patel, J., and Mohapatra, G. P., Some applications of differential subordination to a general class of multivalent functions, Adv. Stud. Contemp. Math. (Pusan) 3 (2001), 1-15.

CENTRAL UNIVERSITY OF RAJASTHAN

16 NAVDURGA COLONY

OPP. HOTEL CLARKS AMER

MALVIYA NAGAR

JAIPUR-302017, RAJASTHAN

INDIA

E-mail: jkp_0007@rediffmail.com
M.P. UNIVERSITY OF AGRICULTURE AND TECHNOLOGY UDAIPUR-313001, RAJASTHAN INDIA

Present Address:

10/11 GANPATI VIHAR, OPPOSITE SECTOR 5

UDAIPUR- 313002, RAJASTHAN

INDIA

E-mail: rkraina_7@hotmail.com 